\title{
Primary diffuse large B-cell lymphoma of the left ureter: A case report
}

\author{
PING CHEN ${ }^{1}$, MAOQING JIANG $^{2}$, YUANWEI LIN $^{2}$, XIANWANG YE $^{2}$, XINZHONG RUAN $^{2}$ and QIULI HUANG ${ }^{2}$ \\ Departments of ${ }^{1}$ Nephrology and ${ }^{2}$ Radiology, Ningbo First Hospital, Ningbo, Zhejiang 315000, P.R. China
}

Received October 6, 2015; Accepted June 13, 2016

DOI: $10.3892 / \operatorname{mco} .2016 .949$

\begin{abstract}
Diffuse large B-cell lymphoma (DLBCL) is a subtype of non-Hodgkin lymphoma occurring in various sites, but rarely involving the ureters. Primary DLBCL is a rare entity. Imaging studies in a 82-year-old male patient revealed left hydronephrosis and an area of nodular soft tissue density in the upper ureteral wall. On enhanced computed tomography scans, the lesion exhibited early enhancement. As the lesion was considered to be malignant, a left nephroureterectomy was performed for the purpose of pathological diagnosis. Histological analysis and immunohistochemistry revealed DLBCL. Since the surgery, the patient has survived for 16 months without evidence of a relapse. Thus, in cases with ureteral stenosis or obstruction for which the cause is uncertain, the possibility of primary lymphoma of the ureter should be considered and further histopathological examination of bioptic samples should be performed as soon as possible.
\end{abstract}

\section{Introduction}

Ureteric involvement by non-Hodgkin lymphoma (NHL) is rare. It is difficult to distinguish ureteral lymphoma from urothelial carcinoma despite using well-established imaging modalities, such as positron emission tomography/computed tomography (PET/CT), antegrade CT pyelography (ACTP) and CT urography (CTU) $(1,2)$. We herein present a case of a 82-year-old male patient with left hydronephrosis and obstructive changes, confirmed following histological analysis and immunohistochemistry to be diffuse large B-cell lymphoma (DLBCL). Therefore, NHL should be included in the differential diagnosis of patients with primary neoplasms of the ureter or metastases to the ureter. In the present case,

Correspondence to: Dr Qiuli Huang, Department of Radiology, Ningbo First Hospital, 59 Liuting Road, Haishu, Ningbo, Zhejiang 315000, P.R. China

E-mail: huajx20@163.com

Key words: ureter, primary diffuse large B-cell lymphoma, computed tomography the patient underwent surgery and has survived for 16 months without evidence of recurrence. The aim of this study was to discuss the diagnosis and treatment of malignant lymphomas of the ureter along with a review of the relevant literature.

\section{Case report}

In March 2014, a 82-year-old Chinese man was admitted to the Ningbo First Hospital (Ningbo, China) with a major complaint of left flank pain for 2 weeks. Physical examination revealed left renal pain on percussion; however, there were no other remarkable findings. The blood routine tests were normal, with a white blood cell count of $4.8 \times 10^{9} / 1$ (neutrophils, $76.6 \%$ ), a hemoglobin level of $12.0 \mathrm{~g} / \mathrm{dl}$ and a platelet count of $123 \times 10^{9} / 1$. There was no systemic inflammation and the C-reactive protein level was $<0.3 \mathrm{mg} / \mathrm{dl}$. The urine cytology and urinalysis findings were normal.

The erect abdominal plain radiograph revealed no abnormalities (Fig. 1). Ultrasonography demonstrated left hydronephrosis. Abdominal computed tomography (CT) images revealed narrowing of the proximal third of the left ureter, with a soft tissue mass of the ureteral wall measuring $\sim 2.0 \times 1.5 \mathrm{~cm}$ (Fig. 2A). The soft tissue ureteral mass was attached to the adjacent left psoas major muscle (Fig. 2A). On enhanced CT scans, the lesion exhibited early and continuous enhancement (Fig. 2B and C). Enlarged lymph nodes were not identified in the retroperitoneum. Left hydronephrosis and obstruction of the left ureter were shown by CTU (Fig. 3). The CT of the chest was normal, without abnormal enlarged mediastinal lymph nodes.

As the lesion was considered to be malignant, left nephroureterectomy was performed. Histological analysis revealed a typical lymphocytosis, mainly of the large-cell type (Fig. 4). On immunohistochemistry, the lesion was positive for leukocyte common antigen, CD79a, CD20, vimentin, CD68 and negative for cytokeratin (CK) 7, CK20, myeloperoxidase, CD43, CD45RD, soluble protein-100, Synaptophysin, human melanoma black 45, melan-A (A103) and carcinoembryonic antigen. These findings were consistent with DLBCL. The bone marrow biopsy showed no involvement by lymphoma. Moreover, the patient underwent gastroscopy, which revealed no abnormalities. Taking into consideration the advanced age of patient, systemic chemotherapy was not administered. Since the surgery, the patient has survived for 16 months without evidence of recurrence. 


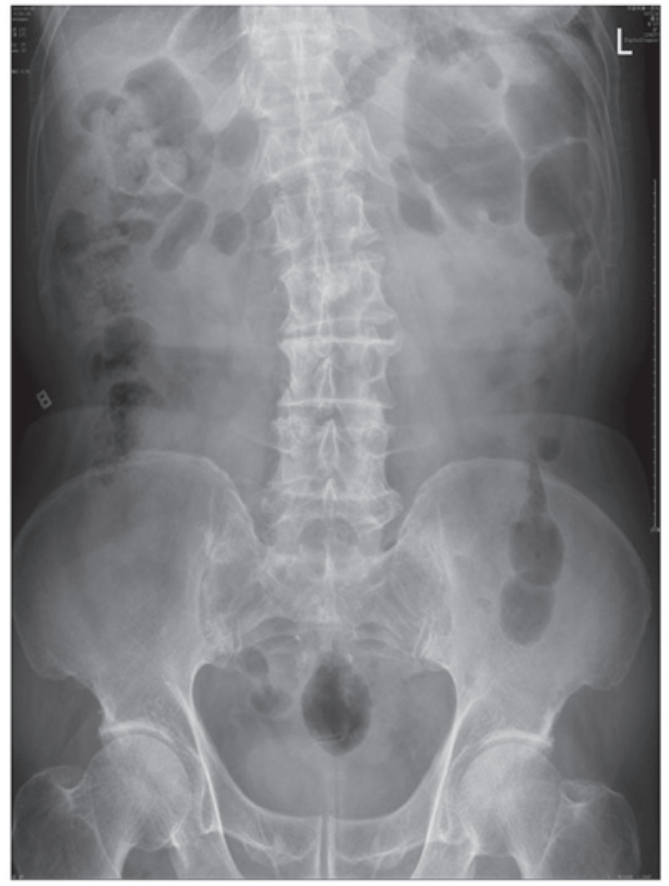

Figure 1. Erect abdominal plain radiograph showing degeneration of the lumbar spine. No other abnormalities, such as ureteral calculi, were observed on either side.
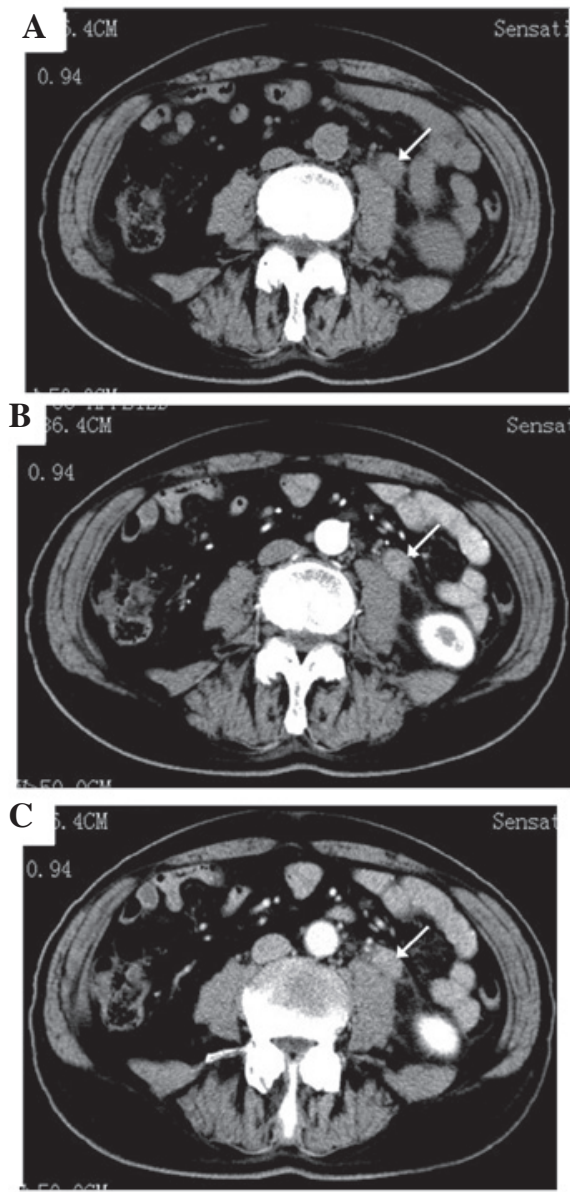

Figure 2. (A) Abdominal computed tomography (CT) images revealed a nodular soft-tissue density area (arrow) in the wall of the proximal third of the left ureter, which was attached to the adjacent left psoas major muscle. On enhanced CT scans, the lesion exhibited early and continuous enhancement in the (B) arterial and (C) venous phase.

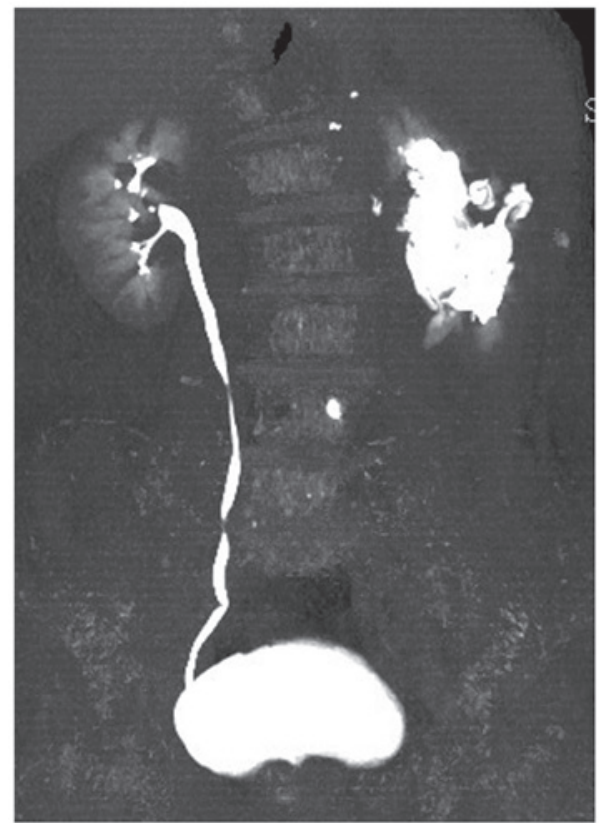

Figure 3. Computed tomography urography showing obstruction of the left ureter and left hydronephrosis.

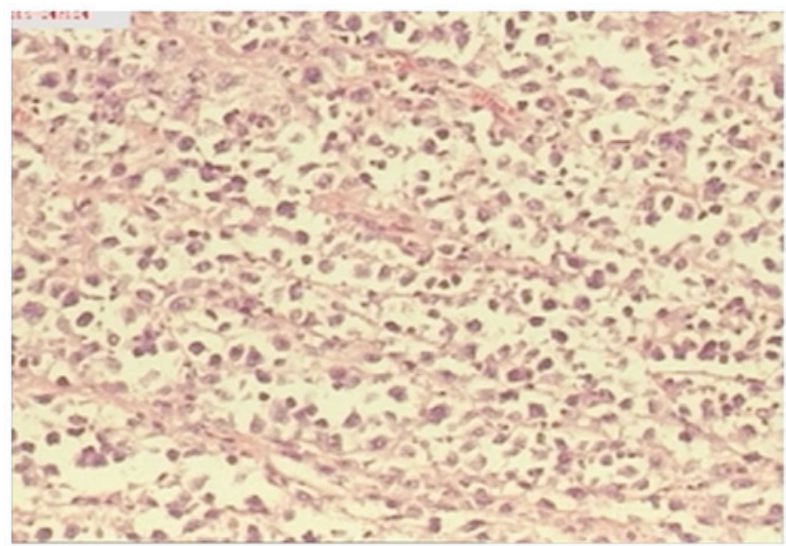

Figure 4. Histological examination of the lesion revealed a typical lymphocytosis, mainly of the large-cell type (hematoxylin and eosin staining; magnification, $\mathrm{x} 100$ )

\section{Discussion}

DLBCL is a common subtype of NHL occurring in various sites, most commonly in the gastrointestinal tract, lungs, salivary glands, thyroid gland, skin, orbits and bones, whereas involvement of the ureters is extremely uncommon (3). Ureteral involvement by lymphoma is usually caused by contiguous spread from the retroperitoneal lymph nodes (4). Overall, 25-40\% of all lymphomas present as primary extranodal NHL (5). It is difficult to distinguish whether ureteric lymphomas are primary or secondary to metastasis, particularly in patients where nodal and extranodal sites are involved (4). In the present case, however, no abnormally enlarged retroperitoneal lymph nodes were identified, and there was no involvement of extranodal sites. Thus, the tumor was likely a primary DLBCL of the left ureter.

Clinical diagnosis is very difficult due to the lack of typical symptoms or particular imaging characteristics. The majority 
of the patients exhibit no evident symptoms or only complain of back pain (6). Certain patients may also present with hematuria (2) or postrenal azotemia (7). In the present case, the patient complained of left flank pain for 2 weeks, with no other abnormal physical signs. Ghersin et al (1) proposed a combination PET/CT scan to more effectively detect and localize malignant lesions. However, differentiating between physiological and pathological uptake due to malignancy using PET alone may be difficult (1). Hashimoto et al (8) suggested that ureteroscopy with biopsy provided more definitive findings compared with radiography for differentiating primary neoplasms from metastases to the ureter. In our patient, the imaging findings were an area of nodular soft tissue density in the upper ureteral wall on CT images, hydronephrosis on CTU images and early enhancement on enhanced CT scans. The final diagnosis was confirmed by nephroureterectomy followed by histopathological examination, which revealed malignant lymphoma of the ureter.

The reported cases of NHL of the ureter vary in terms of treatment and include radiation after partial ureterectomy or chemotherapy. Ruth et al (3) reported on a 50-year-old man with ureteric DLBCL with HIV who received R-CHOP chemotherapy and remained alive at 6 months. Vedovo et al reported on an 82-year-old female patient who underwent radical nephrectomy for a relapsed renal mass and she diagnosed with lymphoplasmacytic MALT lymphoma (9). In the present case, left nephroureterectomy was performed for the purpose of pathological diagnosis. The histological analysis and immunohistochemistry suggested a diagnosis of DLBCL. Clinicians considered that the 82-year-old patient was not likely to tolerate chemotherapy; thus, the patient underwent surgery alone. Following surgery, the patient has survived for 16 months without evidence of recurrence.

In conclusion, although primary DLBCL of the left ureter is extremely rare, it is crucial that it is identified early for diagnostic and therapeutic implications. Our case further confirmed that primary DLBCL may occur in the ureter and should be considered in the differential diagnosis of soft tissue masses involving the ureter. However, as the disease may relapse, a longer follow-up is required prior to suggesting that surgery alone may be curative.

\section{References}

1. Ghersin E, Keidar Z, Eldad DJ, Bar-Shalom R, Fischer D and Halachmi S: Multimodality imaging of direct ureteric involvement in non-Hodgkin's lymphoma using PET/CT, CT urography and antegrade CT pyelography. Br J Radiol 80: e283-e286, 2007.

2. Jaeger CD, McAlvany KL, Zingula SN, Kramer SA and Granberg CF: Diffuse large B-cell lymphoma in an adolescent male presenting as ureteral stricture. Case Rep Radiol 2014: 239345, 2014.

3. Ruth K, El-Amm J, Sotelo T and Aragon-Ching JB: Primary diffuse large B-cell lymphoma of the ureter in a patient with HIV: A case report and review of literature. Clin Lymphoma Myeloma Leuk 13: 324-326, 2013.

4. Salem AB, Nfoussi $\mathrm{H}$ and Kchir N: Ureteral spread of a primary cutaneous diffuse large B-cell lymphoma, leg type. Indian J Urol 30: 222-224, 2014.

5. Krol AD, le Cessie S, Snijder S, Kluin-Nelemans JC, Kluin PM and Noordijk EM: Primary extranodal non-Hodgkin's lymphoma (NHL): The impact of alternative definitions tested in the comprehensive cancer centre west population-based NHL registry. Ann Oncol 14: 131-139, 2003.

6. Ni BW, Zhong L, Wang T and Chen FY: Malignant lymphoma of the ureter: A case report and literature review. Exp Ther Med 7: 1521-1524, 2014.

7. Kubota Y, Kawai A, Tsuchiya T, Kozima K, Yokoi S and Deguchi T: Bilateral primary malignant lymphoma of the ureter. Int J Clin Oncol 12: 482-484, 2007.

8. Hashimoto H, Tsugawa M, Nasu Y, Tsushima T and Kumon H: Primary non-Hodgkin lymphoma of the ureter. BJU Int 83: 148-149, 1999.

9. Vedovo F, Pavan N, Liguori G, Siracusano S, Bussani R and Trombetta C: Primary renal MALToma: A rare differential diagnosis for a recurrent renal mass after primary ablative therapy. Can Urol Assoc J 8: E442-E444, 2014. 